Strong-field ionization in time-dependent density functional theory

This article has been downloaded from IOPscience. Please scroll down to see the full text article.

2008 EPL 8443001

(http://iopscience.iop.org/0295-5075/84/4/43001)

View the table of contents for this issue, or go to the journal homepage for more

Download details:

IP Address: 131.174.17.23

The article was downloaded on 02/03/2012 at 10:40

Please note that terms and conditions apply. 


\title{
Strong-field ionization in time-dependent density functional theory
}

\author{
A. S. DE WiJN ${ }^{1}$, M. LeIN ${ }^{2}$ and S. KÜMMEL ${ }^{1,3}$ \\ ${ }^{1}$ Max-Planck-Institut für Physik komplexer Systeme - Nöthnitzer Strasse 38, D-01187 Dresden, Germany, EU \\ ${ }^{2}$ Institut für Physik, Universität Kassel - D-34132 Kassel, Germany, EU \\ ${ }^{3}$ Physikalisches Institut, Universität Bayreuth - D-95440 Bayreuth, Germany, EU
}

received 18 July 2008; accepted in final form 9 October 2008

published online 10 November 2008

PACS 31.15.ee - Time-dependent density functional theory

PACS 32.80.Rm - Multiphoton ionization and excitation to highly excited states

PACS 71.15. Mb - Density functional theory, local density approximation, gradient and other corrections

\begin{abstract}
Ionization in a strong laser field is a prime example of non-perturbative, correlated electron dynamics. Simulating such processes on a first-principles basis is a major theoretical challenge. We demonstrate that time-dependent density functional theory (TDDFT) on the basis of a newly developed functional for the correlation potential incorporates the relevant electron interaction effects. The central idea of our approach is to exploit information about exact groundstate correlation in approximate time-dependent calculations. The new functional provides an accurate description of the paradigm problem of the double ionization of the Helium atom, showing that TDDFT is a viable tool for strong-field calculations.
\end{abstract}

Copyright (C) EPLA, 2008

Introduction. - The interaction of intense laser pulses with finite electronic systems such as atoms and molecules has developed into a powerful tool for investigating matter on extremely small length and time scales. This has opened routes to microscopically manipulate electronic systems [1-3] and to gain insights into the nature of lightmatter interaction and electronic dynamics [4,5]. Hopes are high that steering chemical reactions and observing electronic dynamics in real time will become standard techniques in the future.

One of the milestones in strong-field physics was the investigation of the double ionization of the He atom. It was demonstrated that in a strong field the electronelectron interaction plays a tremendously more important role than anticipated, as the probability for doubly ionizing the atom is orders of magnitude larger than expected from a sequential process. Thus, non-sequential ionization leads to the famous "knee structure" $[6,7]$ in the intensity dependence of the double-ionization probability. The generally accepted explanation for this effect is the threestep recollision mechanism [8]: in the first step, the atom is ionized; in the second step, the free electron is accelerated over large distances $\left(\approx 100 a_{0}\right.$, where $a_{0}$ is the Bohr radius) by the infrared-frequency field; finally the electron recollides with the ion and kicks out the second electron.
Although the fundamental nature of this process is understood [9-13], quantum-mechanical first-principles calculations that simulate this type of strong-field electron dynamics are extremely difficult due to the fact that a nonperturbative approach is required at the experimentally realized field strengths. Solving the correlated timedependent (TD) Schrödinger equation on the relevant length and time scales is an outstanding computational challenge already for a two-electron system even with today's supercomputers [14]. This is largely due to the fact that $a b$ initio wave function techniques gaining their efficiency from using localized basis sets cannot be used to describe dynamics on a length scale of about $100 a_{0}$.

An alternative quantum-mechanical approach that avoids the immense computational effort associated with using the two- or multi-electron wave function is the time-dependent density functional theory (TDDFT). In this work we show that known difficulties with "standard" TDDFT approaches can be overcome by a new type of exchange correlation functional. Thus, we demonstrate that TDDFT can be a powerful tool for theoretically investigating strong-field processes.

Kohn-Sham theory. - TDDFT in the Kohn-Sham framework [15] relies on reproducing the interacting 
time-dependent density $n(\mathbf{r}, t)$, in principle exactly, by solving [16-19] the time-dependent Kohn-Sham equations

$$
i \hbar \frac{\partial}{\partial t} \varphi_{j}(\mathbf{r}, t)=\left[-\frac{\hbar^{2}}{2 m} \nabla^{2}+v_{\mathrm{KS}}(\mathbf{r}, t)\right] \varphi_{j}(\mathbf{r}, t) .
$$

Here, $\varphi_{j}(\mathbf{r}, t)$ are single-particle orbitals yielding the density $n(\mathbf{r}, t)=\sum_{j=1}^{N}\left|\varphi_{j}(\mathbf{r}, t)\right|^{2}$, and

$$
v_{\mathrm{KS}}(\mathbf{r}, t)=v_{\mathrm{ext}}(\mathbf{r}, t)+v_{\mathrm{H}}(\mathbf{r}, t)+v_{\mathrm{xc}}(\mathbf{r}, t)
$$

is the time-dependent Kohn-Sham potential. It comprises the given external potential $v_{\text {ext }}$, the classical Hartree potential $v_{\mathrm{H}}(\mathbf{r}, t)=e^{2} \int n\left(\mathbf{r}^{\prime}, t\right) /\left|\mathbf{r}-\mathbf{r}^{\prime}\right| \mathrm{d}^{3} r^{\prime}, \quad$ and the exchange correlation (xc) potential $v_{\mathrm{xc}}$. The latter potential is the crucial part of Kohn-Sham TDDFT, because it incorporates all parts of the electron interaction that have not been accounted for in the Hartree potential. As the exact expression for $v_{\mathrm{xc}}$ is unknown, TDDFT in practice has to rely on approximations to the unknown exact xc potential, and this is the point where many problems with TDDFT calculations in the strong-field regime have their origin. Several works have convincingly demonstrated [20-23] that the standard approximations [24,25] for $v_{\mathrm{xc}}$ such as the "Adiabatic Local Density Approximation" (ALDA) do not reproduce hallmark features of interacting electron dynamics in strong fields, such as the above-mentioned knee structure. In fact, their results are quite similar to the results of completely uncorrelated, exchange-only calculations. This has been a long-standing puzzle, in striking contrast to the huge success that TDDFT has in the linear response regime where it has become one of the most widely used methods, e.g., for calculating photoabsorption spectra of molecules.

The xc potential of Kohn-Sham theory. - The success of TDDFT in the linear response regime is largely based on the fact that even relatively simple approximations such as the ALDA lead to a quite reasonable description of the experimentally relevant electronic excitations. Inherent to ALDA or its extensions such as the adiabatic time-dependent generalized gradient approximations are two approximations [24]: i) locality in space, i.e., $v_{\mathrm{xc}}^{\mathrm{ALDA}}$ at a given point $\mathbf{r}$ is determined by the density only at $\mathbf{r}$; ii) locality in time, i.e., $v_{\mathrm{xc}}^{\mathrm{ALDA}}$ at a given time $t$ is determined by the density only at $t$. Generally speaking, both of these approximations can be quite severe, as it is known that the exact $v_{\mathrm{xc}}$ depends non-locally on $\mathbf{r}$ and $t$, i.e., the exact xc potential at a given time $t$ and point $\mathbf{r}$ is a functional of the density at all points in space and all times prior and up to $t$. Thus, the fact that functionals like ALDA fail completely in the description of double ionization could be attributed to different reasons. However, recent work has clarified that the qualitatively wrong description of strong-field ionization is a consequence of a missing step-like structure in the popular xc potential approximations, whereas the missing temporal non-locality only plays a minor role under the typical experimental conditions [26-28]. While the source of the problems has thus been pointed out, it has remained unclear how to non-empirically construct practically useful exchange correlation potentials that have the desired and required feature of a quantitatively correct step structure. In the following we present a way in which this can be achieved.

A new approach to correlation. - The basic concept of our approach is to use information from highly accurate ground-state densities to construct a potential that can be used in non-perturbative TDDFT calculations. Thus, in an indirect way, we exploit a rarely used aspect of the Hohenberg-Kohn theorem [29] which states that the ground-state density uniquely determines the entire Hamiltonian of a system and thus not only the system's ground state, but all of its states. On the practical side the crucial feature of our approximation is that it exhibits a quantitatively correct step-like structure in the correlation potential which is closely related to the famous derivative discontinuity [30,31].

The construction of our approximation is based on the ensemble interpretation of fractional particle numbers that underlies the concept of the derivative discontinuity. For a step-by-step introduction, consider a helium atom in an ionizing laser field which we take as constant for simplicity of the argument. Focus on a region of space around the nucleus, e.g., a sphere of radius $R$, which contains practically all of the ground-state density in the sense that when we integrate the ground-state density over the sphere, we will obtain a number very close to 2. If the ionizing field is switched on, the density will be polarized and part of the density will leave the sphere, being accelerated towards infinity. Now integrating the remaining density over the sphere will lead to a number smaller than 2. More precisely, as a function of time the average number of electrons bound to the nucleus will drop from 2 to 0 if the field is strong enough to ionize both electrons. In ref. [26] it was demonstrated that a step structure builds up in the exact TD electron interaction potential $v_{\mathrm{H}}+v_{\mathrm{xc}}$ as the average electron number drops towards 1 , and that this step structure is decisive for a correct description of the electron dynamics. It was further demonstrated that a quantitatively similar step structure is inherent to the exact ground-state electron interaction potential that corresponds to a ground-state density which integrates to the same average number of electrons as the time-dependent density. In other words, ref. [26] showed that the two following potentials show step structures that are quantitatively very similar to each other: i) the numerically exact TD xc potential obtained from the exact TD density by inverting the TD KohnSham scheme, and ii) the ground-state xc potential for a ground-state density that integrates over the sphere to the same fractional number of electrons. This observation also makes sense physically, because it has long been known [30] that the exact ground-state xc potential as a function of particle number changes discontinuously at 
integers. The step structure seen in the xc potential reflects this discontinuity $[27,31]$.

With these facts in mind the following approximation to the time-dependent xc potential - presented here for the specific case of the Helium ionization - comes to mind naturally. First, calculate the exact ground-state density of $\mathrm{He}$ and of $\mathrm{He}^{+}$(the latter being known analytically) and obtain the exact ground-state Kohn-Sham potential by inversion of the static Kohn-Sham equation. For a twoelectron singlet state as in helium, this is trivial: the orbital is given by $\varphi=\sqrt{n / 2}$, and from the orbital one obtains the potential

$$
v_{\mathrm{KS}}(\mathbf{r})=\frac{\hbar^{2}}{2 m} \frac{\nabla^{2} \varphi}{\varphi}+\mathrm{const}
$$

to within an irrelevant constant. By subtracting the known $v_{\text {ext }}, v_{\mathrm{H}}$ and $v_{\mathrm{x}}=-v_{\mathrm{H}} / 2$, which can easily be calculated for a given density, one obtains the exact ground-state correlation potential

$$
v_{\mathrm{c}}=v_{\mathrm{KS}}-v_{\mathrm{ext}}-v_{\mathrm{H}}-v_{\mathrm{x}} .
$$

This can be used in the solution of the time-dependent Kohn-Sham equation (1), e.g., following the techniques described in [32], for the first numerical propagation step from $t_{\text {ini }}$ to $t=t_{\text {ini }}+\Delta t$, yielding $\varphi(\mathbf{r}, t)$. After the first time step $\Delta t$, the new $v_{\mathrm{KS}}(\mathbf{r}, t)$ (eq. (2)) at the new, advanced time $t$ must be calculated. The potentials $v_{\mathrm{H}}$ and $v_{\mathrm{x}}$ can readily be calculated exactly from the new, propagated density, but the new correlation potential cannot be obtained exactly. However, based on the arguments of the preceeding paragraph, it can be approximated as follows. First, one calculates the effective average number of bound electrons $N(t)$ at $t$. This will be a number less than 2, i.e., $N(t)=1+\epsilon(t)$ with $0<\epsilon<1$ for a reasonable physical excitation and a small time step. Then, construct the exact ground-state ensemble density that corresponds to this particle number according to ref. [30], i.e., calculate

$$
n_{1+\epsilon}(\mathbf{r})=(1-\epsilon) n_{1}(\mathbf{r})+\epsilon n_{2}(\mathbf{r}),
$$

where $n_{1}(\mathbf{r})$ denotes the ground-state density of $\mathrm{He}^{+}$ (which contains one electron) and $n_{2}(\mathbf{r})$ denotes the ground-state density of He. From this ensemble density one can calculate the Kohn-Sham orbital $\varphi_{\mathrm{ens}}=\sqrt{n_{1+\epsilon} /(1+\epsilon)}$ which, when inserted into eqs. (3) and (4), yields the exact atomic ground-state correlation potential $v_{\mathrm{c}}^{N}$ corresponding to the average particle number $N(t)$ at the present time step. The total Kohn-Sham potential which is then employed for the second time step, leading from $t_{\text {ini }}+\Delta t$ to $t_{\text {ini }}+2 \Delta t$, is

$$
v_{\mathrm{KS}}=v_{\mathrm{ext}}+v_{\mathrm{H}}+v_{\mathrm{x}}+v_{\mathrm{c}}^{N},
$$

i.e., the exact time-dependent Hartree and exchange potential are combined with the exact atomic ensemble ground-state correlation potential. In other words, instead of the exact time-dependent correlation potential we use the exact atomic ground-state correlation potential that corresponds to the same average electron number near the nucleus. When $N(t)$ falls below 1 , then the interpolation of eq. (5) has to be taken between the 0- and 1-electron densities (both of which are trivial to deal with exactly).

Let us discuss this approximation on general grounds before we test it for specific examples. Considering the practical side first, it is clear that compared to a simple functional such as ALDA our approach is considerably more involved because it requires very accurate ground-state densities as input and needs the inversion of the static ground-state Kohn-Sham equations. Without doubt, this creates a certain computational burden. However, this burden is quite manageable as ground-state densities can be calculated accurately with quantumchemistry techniques and various methods to efficiently invert the ground-state Kohn-Sham equations for systems of any electron number have been developed [33-35]. Furthermore, the accurate densities need to be calculated only once before the time-dependent calculation and not at every time step. Therefore, the computational load at each time step, which determines the load of the full propagation, is not affected by the computational burden that is associated with obtaining the accurate ground-state densities. It should also be noted that the approximation is not restricted to 2-electron systems, as the principle of linear interpolation on which eq. (5) is based is inherent to all many-electron systems [30]. The major advantage of our approximation is that $v_{\mathrm{H}}$ and $v_{\mathrm{x}}$ are treated exactly while the correlation approximation is defined in a non-empirical way without adjustable parameters yielding a potential with the desired step structure.

As an aside we note that in future work it may be possible to use the same concept to a posteriori improve a given functional, e.g. ALDA, by using a Kohn-Sham potential similar to eq. (6), namely

$$
v_{\mathrm{KS}}=v_{\mathrm{ext}}+v_{\mathrm{H}}+v_{\mathrm{x}}+v_{\mathrm{c}}^{N}+v_{\mathrm{c}}^{\mathrm{ALDA}}-v_{\mathrm{c}}^{N \mathrm{ALDA}} .
$$

Here, $v_{\mathrm{c}}^{N \text { ALDA }}$ denotes the correlation potential of ALDA for the ground-state density corresponding to $N$ bound electrons, as explained in the paragraph surrounding eq. (5).

On the theoretical side it is worthwhile to compare the here-proposed approximation to the "adiabatically exact one". The adiabatically exact Kohn-Sham potential is the uniquely defined single-particle potential which exactly reproduces a given time-dependent density as the solution of a ground-state problem. A procedure which explicitly constructs this adiabatically exact potential has been discussed in ref. [28]. The approximation which we propose here is not the adiabatically exact one but can be termed " $N$-adiabatic", because the quantity that is taken to be exactly the same in the time-dependent and the ground-state problem is not the whole density, but only the average number of particles near the nucleus, $N$. Thus, in addition to the approximation of adiabaticity, i.e., the neglect of memory effects in $v_{\mathrm{c}}$, our present approximation 
takes two further steps: It neglects the effect that a polarization of the density through the external field has on the correlation potential, and it neglects the effects that the density far away from the nucleus has on the correlation potential. Phrasing this the other way round we can say that the physical approximation inherent to our functional is that correlation plays a role mainly for the description of how electrons are ionized and interact close to the nucleus.

A paradigm test: strong-field double ionization. - As explained in the first two sections of this manuscript, a critical test for whether these approximations are justified is to employ the new functional to calculate the strongfield ionization of helium. All of the usually employed density functionals fail badly in this test $[20,22,23]$. This is also a particularly hard problem to solve with TDDFT, because density functional theory usually works better with a large number of electrons than with a small number, and in this sense the helium problem is the hardest test case one can find.

For evaluating a new approach we need well-defined and precise results to compare with. In our case, the ultimate benchmark would be the solution of the TD Schrödinger equation, which, as explained above, is not easily available for the true, three-dimensional helium atom. However, for our purposes a suitable alternative is available in the form of the widely used one-dimensional model of the helium atom. This model has been shown to incorporate the relevant physical effects $[10,20,21,36,37]$ and is computationally tractable enough to allow for a full solution of the TD Schrödinger equation. The model is described by the Hamiltonian

$$
H=\sum_{j=1,2}\left(\frac{p_{j}^{2}}{2 m}+v_{\mathrm{ext}}\left(z_{j}, t\right)\right)+W\left(z_{1}-z_{2}\right),
$$

where $z_{1}, z_{2}$ are the spatial electron coordinates, $p_{1}, p_{2}$, their momenta, and $W(z)=e^{2} / \sqrt{z^{2}+1}$ is the soft-core Coulomb interaction. The external potential $v_{\text {ext }}(z, t)=$ $-2 W(z)+e z E(t)$ comprises the nuclear attraction and the external field of the laser $E(t)$. We take the wave function to be a spin singlet in accordance with the situation in real helium. We do not fit the value of the softening parameter to any specific numbers but use the canonical value of 1 in $W$, as our aim is a general comparison. The ground-state and time-dependent wave function of this model can be obtained accurately with the methods described in [32]. This gives the reference results. The time-dependent Kohn-Sham equation eq. (1), is solved under the same conditions (spin singlet, same external field, same numerical algorithms) with eq. (6) for the Kohn-Sham potential.

With this setup we first investigate a simple situation in order to obtain an as transparent picture as possible. The He atom is initially in its ground state and then subjected to an ionizing electrical field that is ramped over

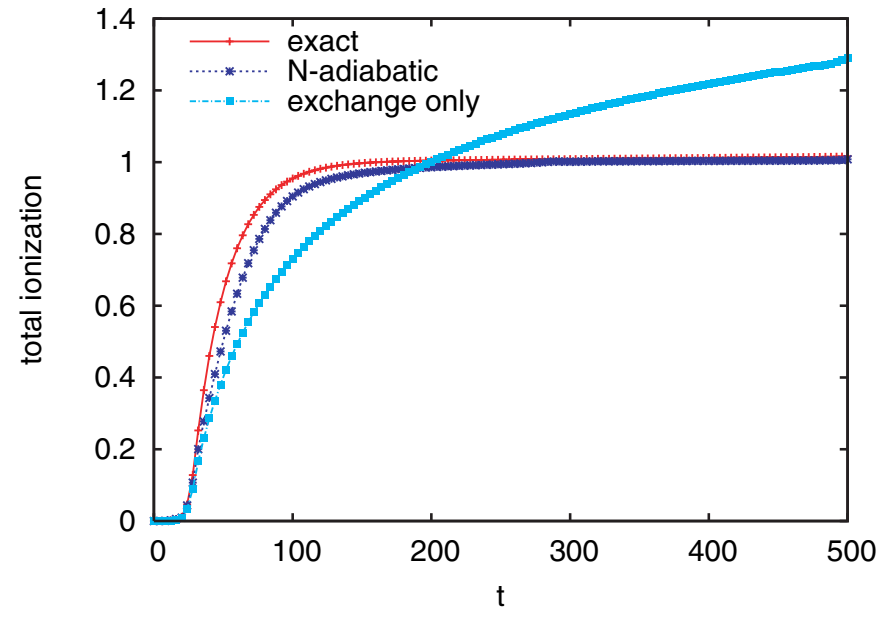

Fig. 1: (Colour on-line) Total ionization as a function of time (in a.u.) of the helium model, eq. (8), in a strong field. The field was linearly ramped up from 0 to 0.1677 a.u. between $t=0$ and $t=24$ a.u. Shown are i) the exact results from a numerical solution of the TD Schrödinger equation (red crosses), ii) the $N$-adiabatic approximation of eq. (6) (blue stars), and iii) an exchange-only calculation (cyan squares). The exchange-only calculation completely misses the leveling-off of the ionization around $N=1$ which is seen in the exact calculation and the $N$-adiabatic one.

a time of 24 atomic units (a.u.) up from 0 to a maximum value of 0.1677 a.u., corresponding to the peak amplitude of a pulse with an intensity of $9.9 \times 10^{14} \mathrm{~W} / \mathrm{cm}^{2}$. Our calculations are performed on numerical grids and use absorbing boundary conditions. The grids consist of 4096 points for each spatial coordinate at a grid separation of 0.4 a.u. and the time step size is 0.0333 a.u. For other details, see [32].

The observable that we investigate is the average number of escaped electrons $N(t)-N(t=0)$, where

$$
N(t)=\int_{-R}^{R} n(z, t) \mathrm{d} z
$$

corresponds to the number of electrons close to the nucleus (we choose $R=5 a_{0}$ ) [21]. We refer to the number of escaped electrons as the total ionization (which is different from the total ionization probability). Figure 1 shows that the $N$-adiabatic approximation reproduces the exact curve quite accurately. It also shows that taking into account correlation effects is important: the exchange-only calculation is qualitatively in error, as it underestimates ionization initially (i.e., for $t \lesssim 190$ a.u.) and considerably overestimates it at later times, leading to a total ionization that is seriously in error.

Backed by this encouraging result one can proceed to the real problem of investigating the double ionization in a realistic, short, pulsed laser field. We choose a pulse at $780 \mathrm{~nm}$ wavelength (which is used in many experiments). Our pulse shape is trapezoidal with 2-cycle turn-on and turn-off times and 6 cycles total pulse duration. With these 


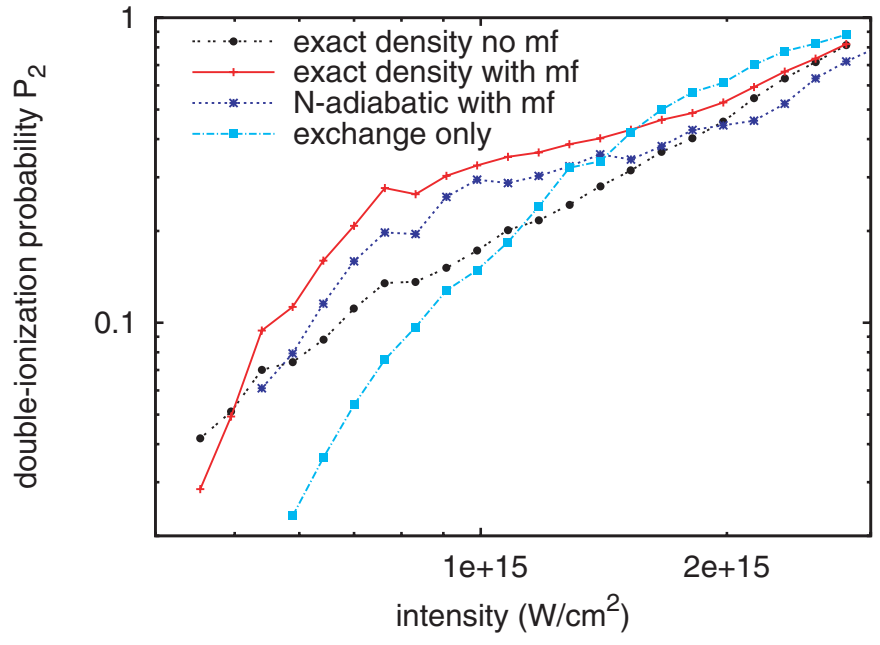

Fig. 2: (Colour on-line) Double-ionization probability as a function of laser intensity for the helium model atom using different approximations. Comparing the curves labeled "exact density with $\mathrm{mf}$ ", " $N$-adiabatic with $\mathrm{mf}$ ", and "exchange only" directly shows the influence of the approximation made for $v_{\mathrm{xc}}$ as all three curves are based on different densities but the same mean-field expression for $P_{2}$ (see main text for further explanation). Labeling of curves is as in fig. 1. The curve labeled "exact density no $\mathrm{mf}$ " shows the exact $P_{2}$, i.e., the one that is obtained from the exact density without making the mean-field approximation for $P_{2}$. This curve shows the influence of correlation in the functional for $P_{2}$.

parameters we calculate the double-ionization probability $P_{2}$ as a function of laser intensity, i.e., we perform a series of calculations with intensities ranging from $4.52 \times 10^{14}$ to $2.80 \times 10^{15} \mathrm{~W} / \mathrm{cm}^{2}$.

When comparing the results of the three different calculations we have to take into account that contrary to the total ionization the double-ionization probability is not known as an exact functional of the density. Thus, in order to avoid secondary errors and a possibly misguided comparison, we compare all results on the same basis by using the mean-field definition of the double-ionization probability [22]. I.e., the density obtained from the numerical solution of the TD-Schrödinger equation (our "exact" benchmark), the one obtained from the TD Kohn-Sham scheme with the $N$-adiabatic potential, and the one from a TD exchange-only calculation are all inserted in the same density-dependent mean-field expression $P_{2, \mathrm{mf}}=$ $\left(1-N\left(t_{\text {final }}\right) / 2\right)^{2}$. In this way we ensure that our results for $P_{2}$ reflect the accuracy of our correlation potential without masking of inaccuracies or additional errors from other approximations.

However, it must be noted that a comparison based on the mean-field expression for $P_{2}$ neglects the fact that also $P_{2}$ is a functional of the density in which effects beyond the mean-field approximation play a prominent role $[21,22,38-40]$. This can be seen in fig. 2 by comparing the red crosses (exact density used together with the mean-field expression for $P_{2}$ ) to the black circles (exact density used together with the exact expression for $P_{2}$ ). Therefore, a density-functional-based calculation aiming at comparison to experiment must use an expression for $P_{2}$ that goes beyond the mean-field approximation, e.g., the one from ref. [40]. However, the focus of our work here is to obtain an improved approximation for $v_{\mathrm{xc}}$, and in order to see the effects which $v_{\mathrm{xc}}$ has on the dynamics, a comparison based on the one-electron density, as it is provided by the mean-field expression for $P_{2}$, is sufficient.

Figure 2 shows the results of our calculations. The $N$-adiabatic approximation somewhat underestimates $P_{2}$ (compared to the exact density with mean field) consistently for all intensities and this is no surprise in view of the above discussion and fig. 1. However, apart from this the approximation reproduces the exact curve very well and correctly shows the overall trend as well as characteristic features, e.g., the turnover at $7.6 \times 10^{14} \mathrm{~W} / \mathrm{cm}^{2}$. The great improvement brought about by the correlation potential is seen by comparing with the exchange-only results. The latter show hardly any structure at all, yielding an overall smooth curve that does not show the knee structure. Thus, our calculation shows that the $N$-adiabatic approximation yields a very useful description of the relevant correlation effects, greatly improving over previous TDDFT approximations.

Conclusion and outlook. - In this manuscript we have demonstrated that TDDFT in the time-dependent Kohn-Sham framework can yield a reliable description of electron dynamics in strong fields. The key to this success is taking into account the step-like structure which is the signature of the derivative discontinuity in the xc potential. To this end, we have presented an approximation for the time-dependent correlation potential that makes use of information obtained from accurate ground-state densities. TD Kohn-Sham calculations using this approximation reproduce the knee structure in the intensity dependence of the helium double ionization. Thus, TDDFT appears as a promising route for studying strong-field phenomena.

SK acknowledges financial support by the Deutsche Forschungsgemeinschaft and stimulating discussion with M. Thiele and S. Wüstner. ASdW and SK are grateful for the hospitality of the MPI-PKS.

\section{REFERENCES}

[1] Horn C., Wollenhaupt M., Krug M., Baumert T., De Nalda R. and Bañares L., Phys. Rev. A, 73 (2006) 031401(R).

[2] Brixner T., Damrauer N. H., Niklaus P. and Gerber G., Nature, 414 (2001) 57.

[3] DeSchryver Frans C. et al. (Editors), Femtochemistry (WILEY-VCH, Weinheim) 2001.

[4] Corkum P. B. and Krausz F., Nat. Phys., 3 (2007) 381. 
[5] Lein M., J. Phys. B, 40 (2007) R135.

[6] Fittinghoff D. N., Bolton P. R., Chang B. and Kulander K. C., Phys. Rev. Lett., 69 (1992) 2642.

[7] Walker B. et al., Phys. Rev. Lett., 73 (1994) 1227.

[8] Corkum P. B., Phys. Rev. Lett., 71 (1993) 1994.

[9] Becker A. and Faisal F. H. M., J. Phys. B, 29 (1996) L197.

[10] Liu W.-C., Eberly J. H., Haan S. L. and Grobe R., Phys. Rev. Lett., 83 (1999) 520.

[11] Dundas D., Taylor K. T., Parker J. S. and Smyth E. S., J. Phys. B, 32 (1999) L231.

[12] Moshammer R. et al., Phys. Rev. Lett., 84 (2000) 447.

[13] Weber T. et al., Nature (London), 405 (2000) 658.

[14] Parker J. S., Doherty B. J. S., Meharg K. J. and TAYlOR K. T., J. Phys. B, 36 (2003) L393.

[15] Runge E. and Gross E. K. U., Phys. Rev. Lett., 52 (1984) 997.

[16] Dey B. K. and DeB B. M., Int. J. Quantum Chem., 70 (1998) 441.

[17] Ullrich C. A. U., Reinhard P.-G. and Suraud E., J. Phys. B, 31 (1998) 1871.

[18] Yabana K. and Bertsch G. F., Phys. Rev. A, 40 (1999) 3809.

[19] Chu S., J. Chem. Phys., 123 (2005) 062207.

[20] Bauer D., Phys. Rev. A, 56 (1997) 3028.

[21] Lappas D. G. and van Leeuwen R., J. Phys. B, 31 (1998) L249.

[22] Petersilka M. and Gross E. K. U., Laser Phys., 9 (1999) 105

[23] Bauer D. and Ceccherini F., Opt. Express, 8 (2001) 377.

[24] Gross E. K. U., Dobson J. F. and Petersilka M., Density Functional Theory II, edited by NALEWAJSKI R. F., Top. Curr. Chem., Vol. 181 (Springer, Berlin) 1996, p. 81.
[25] Ullrich C. A., Erhard S. and Gross E. K. U., Super Intense Laser Atom Physics IV, edited by FEDORov M. V., Nato ASI Ser., Vol. 3/13 (Kluwer, Dordrecht) 1996, p. 267.

[26] Lein M. and Kümmel S., Phys. Rev. Lett., 94 (2005) 143003.

[27] Mundt M. and Kümmel S., Phys. Rev. Lett., 95 (2005) 203004.

[28] Thiele M., Gross E. K. U. and Kümmel S., Phys. Rev. Lett., 100 (2008) 153004.

[29] Hohenberg P. and Kohn W., Phys. Rev., 136 (1964) B864.

[30] Perdew J. P., Parr R. G., Levy M. and Balduz J. L. jr., Phys. Rev. Lett., 49 (1982) 1691.

[31] Kümmel S. and KroniK L., Rev. Mod. Phys., 80 (2008) 3 .

[32] De Wijn A. S., Kümmel S. and Lein M., J. Comput. Phys., 226 (2007) 89.

[33] Wang Y. and Parr R. G., Phys. Rev. A, 47 (1993) R1591.

[34] Umrigar C. J. and Gonze X., Phys. Rev. A, 50 (1994) 3827.

[35] Gritsenko O. V., van Leeuwen R. and Baerends J. E., Phys. Rev. A, 52 (1995) 1870.

[36] Lappas D. G., Sanpera A., Watson J. B., Burnett K., Knight P. L., Grobe R. and Eberly J. H., J. Phys. $B, 29$ (1996) L619.

[37] Lein M., Gross E. K. U. and Engel V., Phys. Rev. Lett., 85 (2000) 4707.

[38] Bandrauk A. D. and Lu H., J. Phys. B, 38 (2005) 2529.

[39] Bandrauk A. D. and Lu H., J. Mod. Opt., 53 (2006) 35 .

[40] Wilken F. and Bauer D., Phys. Rev. Lett., 97 (2006) 203001. 